\title{
Finding Genetic Risk Factors of Gestational Diabetes
}

\author{
Soo Heon Kwak ${ }^{1}$, Hak C. Jang ${ }^{2,3}$, Kyong Soo Park ${ }^{1,2,4 *}$ \\ ${ }^{1}$ Department of Internal Medicine, Seoul National University Hospital, Seoul 110-744, Korea, \\ ${ }^{2}$ Department of Internal Medicine, Seoul National University College of Medicine, Seoul 110-799, Korea, \\ ${ }^{3}$ Department of Internal Medicine, Seoul National University Bundang Hospital, Seongnam 463-707, Korea, \\ ${ }^{4}$ WCU Department of Molecular Medicine and Biopharmaceutical Sciences, Graduate School of Convergence Science \\ and Technology and College of Medicine, Seoul National University, Seoul 110-744, Korea
}

\begin{abstract}
Gestational diabetes mellitus (GDM) is a complex metabolic disorder of pregnancy that is suspected to have a strong genetic predisposition. It is associated with poor perinatal outcome, and both GDM women and their offspring are at increased risk of future development of type 2 diabetes mellitus (T2DM). During the past several years, there has been progress in finding the genetic risk factors of GDM in relation to T2DM. Some of the genetic variants that were proven to be significantly associated with T2DM are also genetic risk factors of GDM. Recently, a genome-wide association study of GDM was performed and reported that genetic variants in CDKAL1 and MTNR1B were associated with GDM at a genome-wide significance level. Current investigations using next-generation sequencing will improve our insight into the pathophysiology of GDM. It would be important to know whether genetic information revealed from these studies could improve our prediction of GDM and the future development of T2DM. We hope further research on the genetics of GDM would ultimately lead us to personalized genomic medicine and improved patient care.
\end{abstract}

Keywords: genetics, gestational diabetes, type 2 diabetes

\section{Introduction}

Gestational diabetes mellitus (GDM) is defined as abnormal glucose tolerance that is first identified or diagnosed during pregnancy [1]. It is estimated that approximately $2 \%$ to $5 \%$ of all pregnancies in Korean women are complicated by GDM [2]. The clinical significance of GDM is that it increases the risk of adverse pregnancy outcomes [3]. In a recent large-scale multinational prospective study, increased maternal glucose concentration during pregnancy was significantly associated with increased neonatal birth weight, primary cesarean delivery, neonatal hypoglycemia, and increased placental C-peptide levels [4]. After parturition, about $15 \%$ of GDM women had persistent diabetes at an early postpartum period of 2 months [5]. GDM is also reported to recur at a frequency of $45 \%$ in subsequent pregnancies [6]. Women with a previous history of GDM are at increased risk of future development of type 2 diabetes mellitus (T2DM). The risk of T2DM is 3.5 times greater in women with a history of GDM compared to the general population in Koreans [7]. In addition, offspring of GDM women are also at risk of developing obesity and T2DM [8]. The incidence of GDM is expected to rise, as it will parallel the increasing rate of obesity and T2DM. Furthermore, the recent recommendation from the International Association of Diabetes and Pregnancy Study Group has lowered the diagnostic threshold of GDM and is expected to increase the incidence of GDM [9].

\section{Heritability of GDM}

GDM is thought to have strong heritability. GDM women have an increased positive family history of T2DM. Compared to pregnant women with normal glucose tolerance, GDM women have a significantly greater parental history of T2DM (13.2\% vs. $30.1 \%, \mathrm{p}<0.001)$ [10]. In addition, both GDM women and their offspring are at increased risk of future development of T2DM. The heritability estimate of 
T2DM was reported to be quite high $\left(h^{2}=0.69\right)$ in a recent study performed in Europeans [11]. These findings are indirect evidence that GDM has familial tendency. However, there is no study that has specifically evaluated the heritability of GDM using familial clustering or twins [12]. It would be important to estimate the heritability of GDM and compare it with that of T2DM in women.

\section{Pathophysiological Similarities of GDM and T2DM}

During normal pregnancy, women experience increased adiposity and weight gain, which begin near mid-pregnancy and progress throughout the third trimester [13]. In this period, insulin resistance ensues as a consequence of multiple factors, including increased production of placental growth hormone, estrogen, and tumor necrosis factor $\alpha[14$, 15]. Pregnant women with normal glucose tolerance can increase their $\beta$-cell insulin secretion in response to this increased insulin resistance during pregnancy. Although the mechanism of increased $\beta$-cell insulin secretion during pregnancy is not fully understood, it is reported that prolactin, which increases during pregnancy, can repress islet menin levels and stimulate $\beta$-cell proliferation in mice [16]. In addition, recent reports suggest that $\beta$-cell serotonin signaling is also a major determinant of $\beta$-cell mass during pregnancy [17]. It has been suggested from several clinical studies that GDM women have limited insulin secretion capacity that cannot compensate for the increased insulin resistance [13]. Similar to GDM, T2DM is also characterized by relative deficiency in insulin secretion in the face of increased insulin resistance. Various factors, including increased age, obesity, high-fat diet, and sedentary lifestyle, can induce insulin resistance, and those who do not have sufficient $\beta$-cell insulin secretory capacity are more likely to develop T2DM [13]. Therefore, it is said that a large proportion of GDM women are experiencing future T2DM in advance.

\section{Association of T2DM Genetic Variants in GDM}

Based on the findings that GDM women are at high risk of T2DM and both GDM and T2DM share similar pathophysiologies, it is reasonable to assume that they might also share similar genetic risk factors. Soon after the initial reports of T2DM genome-wide association (GWA) studies [18-21], several studies investigated whether genetic variants that were identified through GWA studies of T2DM were also associated with the risk of GDM [22, 23]. Cho et al. [22] genotyped variants in or near CDKAL1, CDKN2A/2B, FTO, HHEX, IGF2BP2, SLC3OA8, TCF7L2, KCNJ11, and $P P A R G$ in 869 GDM women and 632 carefully selected non-

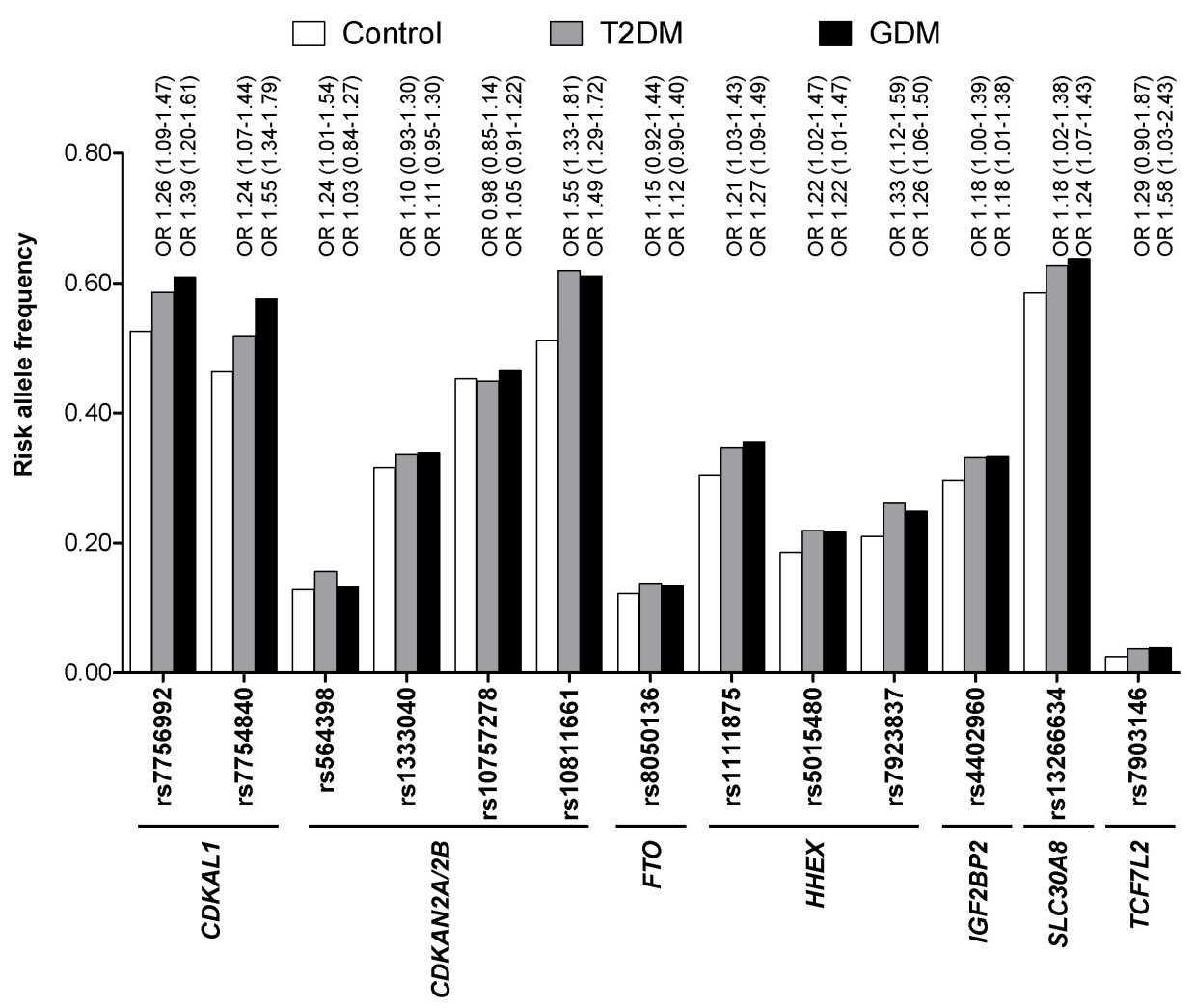

Fig. 1. Comparison of risk allele frequencies of known type 2 diabetes mellitus (T2DM) genetic variants among a control group $(n=632$, men : women $=287: 345)$, T2DM group $(\mathrm{n}$ $=761$, men : women $=354: 407$ ), and gestational diabetes mellitus (GDM) group $(n=869)$ in Koreans. There is an increasing trend of risk allele frequencies from controls to T2DM and from T2DM to GDM. The odds ratio $(\mathrm{OR})$ and $95 \%$ confidence interval of the risk variants for risk of T2DM and GDM are shown above the bars. 
diabetic control subjects. They found that genetic variants in CDKAL1 and CDKN2A/2B were highly associated with the risk of GDM $\left(\mathrm{p}<1 \times 10^{-6}\right)$. In addition, variants in HHEX, IGF2BP2, SLC30A8, and TCF7L2 were all nominally associated with GDM ( $\mathrm{p}<0.05)$. Among a total of 18 genetic variants studied, 9 reached a nominal significance level $(\mathrm{p}<$ 0.05). In Fig. 1, the risk allele frequency as well as the odds ratios of known T2DM variants are compared among a control group $(n=632$, men $:$ women $=287: 345)$, T2DM group ( $\mathrm{n}=761$, men : women $=354: 407)$, and GDM group $(n=869)$ in Koreans $[22,24]$. For most of the variants, there was an increasing trend of risk allele frequencies from control to T2DM and from T2DM to GDM. Lauenborg et al. [23] also found that variants in TCF7L2, CDKAL1, and TCF2 were significantly associated with the risk of GDM in Europeans, consisting of 283 GDM women and 2,446 glucose-tolerant control women. Variants in KCNQ1, which were first identified in an East Asian T2DM GWA study, were also significantly associated with the risk of GDM in Koreans ( $p<0.05$ ) [25]. Recently, it was reported that TPH1 and $H T R 2 B$ play a crucial role in regulating pancreatic $\beta$-cell mass during pregnancy in a mouse model and that their genetic alterations could result in GDM [17]. A total 6 genetic variants in HTR2B and 11 variants in TPH1 were identified and genotyped in Korean GDM women and control subjects [26]. Although there were no significant associations of these variants with the risk of GDM, they were associated with measures of obesity and weight gain during pregnancy [26].

\section{GWA Study of GDM}

Following these candidate approach studies was a twostaged GWA study that was performed in Korean GDM women [27]. A total of 468 GDM women and 1,242 nondiabetic control women were genotyped using Affymetrix Genome-Wide Human SNP Array 5.0. Variants that passed the prespecified $\mathrm{p}$-value threshold in the stage 1 genome scan were further genotyped in 931 GDM women and 783 nondiabetic control women. Two variants, one located in an intron of CDKAL1 (rs7754840) and one near MTNR1B (rs10830962), were associated with a risk of GDM at a genome-wide significance level $\left(\mathrm{p}=6.65 \times 10^{-16}\right.$ and $\mathrm{p}=$ $2.49 \times 10^{-13}$, respectively). This was the first GWA study investigating the genetic risk factors of GDM. Although variants in MTNR1B have been previously reported to be associated with increased fasting glucose levels, the study was the first to report that MTNR1B variants are associated with GDM at a genome-wide significance level. One of the limitations was that it was not sufficiently powered to find truly novel genetic variants that were only specific in GDM.
It should be noted that current GWA and GWA meta-analysis performed in T2DM recruits more than 100,000 cases and controls [28]. One of the interesting findings was that genetic variants of T2DM were enriched in GDM subjects. Among the 34 confirmed T2DM genetic variants, 8 were associated with a risk of GDM [27]. In addition, when the $\beta$-coefficients (or odds ratio) of the variants derived from the logistic regression were compared between GDM and $\mathrm{T} 2 \mathrm{DM}$, there was a significant positive correlation of $\beta$-coefficients between the two [27]. These findings suggest that GDM and T2DM might share similar genetic backgrounds, at least in part.

\section{Perspectives in Genetic Studies of GDM}

GWA studies have opened a new era in diabetes research. Our knowledge on the genetic predisposition of GDM as well as T2DM is expected to increase even faster as next-generation sequencing technology is applied to this field. There should be even larger GWA studies on GDM, and GWA meta-analyses should be available. In this way, we could find variants that have smaller effect sizes but are more specific to GDM than T2DM. In order to understand the genetic determinants of glucose and insulin concentration during pregnancy, genetic associations regarding these quantitative traits should be investigated. A fast way is to see whether genetic variants that are known to affect glucose or insulin concentration in the normal population also affect glucose or insulin concentration during pregnancy [29]. In addition, a GWA study on glucose or insulin concentration during pregnancy should also be helpful. In this way, we would be able to better understand the pathophysiology of GDM.

By definition, GDM encompasses women with pre-exiting T2DM, maturity onset diabetes of the young (MODY), or even type 1 diabetes patients that were not diagnosed previously. It would be important to know the proportion of MODY patients that constitute GDM. In particular, about $15 \%$ of GDM patients remain diabetic at early postpartum periods, and a significant portion of these subjects might fall into the category of MODY. The contribution of genetic variants known to cause MODY, such as GCK and HNF1A, in GDM has been reviewed in recent literature [30]. However, whole-exome sequencing will provide us with better bird's eye view on the contribution of MODY genes in GDM. In addition, it might be able to find novel MODY genes in those who have persistent diabetes after GDM pregnancy and also have a strong family history of diabetes.

One of the first steps in translating the genetic information into clinical practice would be to predict the future development of T2DM in GDM women. GDM women are at particularly high risk of developing T2DM and require 
preventive measures and early screening of T2DM. Genetic information might improve our prediction of T2DM in women with a history of GDM. This is an area of active research [31,32], and we are looking forward to studies that use genotype risk scores in predicting T2DM in GDM women. A similar approach could also be applied in predicting GDM, as more genetic variants associated with GDM are expected to be revealed.

The functional consequences of the current common genetic variants identified through GWA studies of GDM are not well understood yet. It is not known whether they are markers in linkage disequilibrium with nearby causal variants or whether they have unknown but relevant functional roles. Next-generation sequencing might give answers to these questions, but a huge number of samples and much effort will be required.

\section{Concluding Remarks}

GDM women are at increased risk of developing T2DM and have familial clustering of T2DM. A common pathophysiology that is shared by GDM and T2DM is impaired compensatory increase in insulin secretion to overcome the increased insulin resistance. The GWA study of GDM enabled us to investigate the common genetic risk factors of GDM, and it revealed that GDM and T2DM share similar genetic backgrounds, at least in part (Fig. 2). Although this information has significantly improved our insight in the pathogenesis of GDM, there are more unanswered questions

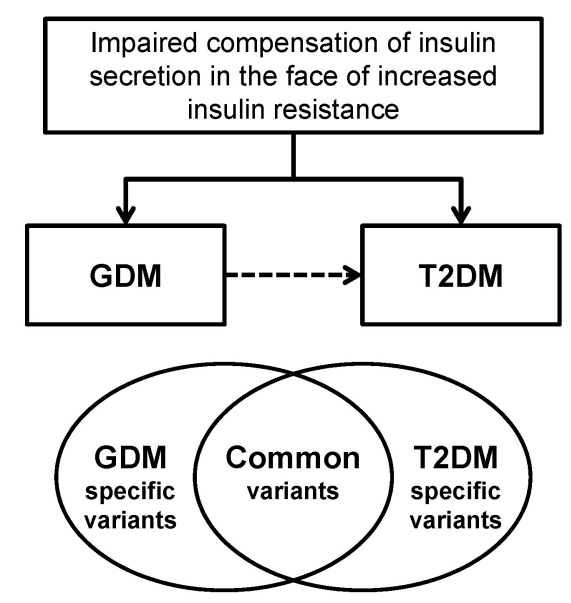

Fig. 2. Gestational diabetes mellitus (GDM) and type 2 diabetes mellitus (T2DM) have similar pathophysiologies of impaired compensation of insulin secretion in the face of increased insulin resistance. GDM women have increased familial clustering of T2DM and are at high risk of developing T2DM. Using genetic association studies, it has been shown that GDM and T2DM share similar genetic backgrounds, at least in part. remaining that should be explicitly expressed. Using the technology of next-generation sequencing, it is expected that more causal, rare variants will be identified. This genetic information would ultimately improve our prediction of GDM and future T2DM. In addition, we hope that personalized genomic medicine could be available using the advances in the genetics of GDM and T2DM.

\section{Acknowledgments}

This work was supported by the National Project for Personalized Genomic Medicine, Ministry for Health \& Welfare (grant no. A111218-12-GM01) and the Korea Healthcare Technology R \& D Project, Ministry of Health and Welfare, Republic of Korea (grant no. A111362).

\section{References}

1. Metzger BE. Summary and recommendations of the Third International Workshop-Conference on Gestational Diabetes Mellitus. Diabetes 1991;40 Suppl 2:197-201.

2. Jang HC. Gestational diabetes in Korea: incidence and risk factors of diabetes in women with previous gestational diabetes. Diabetes Metab J 2011;35:1-7.

3. Jovanovic L, Pettitt DJ. Gestational diabetes mellitus. JAMA 2001;286:2516-2518.

4. HAPO Study Cooperative Research Group, Metzger BE, Lowe LP, Dyer AR, Trimble ER, Chaovarindr U, et al. Hyperglycemia and adverse pregnancy outcomes. $N$ Engl J Med 2008;358: 1991-2002.

5. Jang HC, Yim CH, Han KO, Yoon HK, Han IK, Kim MY, et al. Gestational diabetes mellitus in Korea: prevalence and prediction of glucose intolerance at early postpartum. Diabetes Res Clin Pract 2003;61:117-124.

6. Kwak SH, Kim HS, Choi SH, Lim S, Cho YM, Park KS, et al. Subsequent pregnancy after gestational diabetes mellitus: frequency and risk factors for recurrence in Korean women. Diabetes Care 2008;31:1867-1871.

7. Lee H, Jang HC, Park HK, Metzger BE, Cho NH. Prevalence of type 2 diabetes among women with a previous history of gestational diabetes mellitus. Diabetes Res Clin Pract 2008;81: 124-129.

8. Clausen TD, Mathiesen ER, Hansen T, Pedersen O, Jensen DM, Lauenborg J, et al. Overweight and the metabolic syndrome in adult offspring of women with diet-treated gestational diabetes mellitus or type 1 diabetes. J Clin Endocrinol Metab 2009;94:2464-2470.

9. International Association of Diabetes and Pregnancy Study Groups Consensus Panel, Metzger BE, Gabbe SG, Persson B, Buchanan TA, Catalano PA, et al. International association of diabetes and pregnancy study groups recommendations on the diagnosis and classification of hyperglycemia in pregnancy. Diabetes Care 2010;33:676-682.

10. Jang HC, Min HK, Lee HK, Cho NH, Metzger BE. Short stature in Korean women: a contribution to the multifactorial predis- 
position to gestational diabetes mellitus. Diabetologia 1998; 41:778-783.

11. Almgren P, Lehtovirta M, Isomaa B, Sarelin L, Taskinen MR, Lyssenko V, et al. Heritability and familiality of type 2 diabetes and related quantitative traits in the Botnia Study. Diabetologia 2011;54:2811-2819.

12. Watanabe RM, Black MH, Xiang AH, Allayee H, Lawrence JM, Buchanan TA. Genetics of gestational diabetes mellitus and type 2 diabetes. Diabetes Care 2007;30 Suppl 2:S134-S140.

13. Buchanan TA, Xiang AH. Gestational diabetes mellitus. J Clin Invest 2005;115:485-491.

14. Di Cianni G, Miccoli R, Volpe L, Lencioni C, Del Prato S. Intermediate metabolism in normal pregnancy and in gestational diabetes. Diabetes Metab Res Rev 2003;19:259-270.

15. Barbour LA, McCurdy CE, Hernandez TL, Kirwan JP, Catalano PM, Friedman JE. Cellular mechanisms for insulin resistance in normal pregnancy and gestational diabetes. Diabetes Care 2007;30 Suppl 2:S112-S119.

16. Karnik SK, Chen H, McLean GW, Heit JJ, Gu X, Zhang AY, et al. Menin controls growth of pancreatic beta-cells in pregnant mice and promotes gestational diabetes mellitus. Science 2007; 318:806-809.

17. Kim H, Toyofuku Y, Lynn FC, Chak E, Uchida T, Mizukami H, et al. Serotonin regulates pancreatic beta cell mass during pregnancy. Nat Med 2010;16:804-808.

18. Sladek R, Rocheleau G, Rung J, Dina C, Shen L, Serre D, et al. A genome-wide association study identifies novel risk loci for type 2 diabetes. Nature 2007;445:881-885.

19. Scott LJ, Mohlke KL, Bonnycastle LL, Willer CJ, Li Y, Duren $\mathrm{WL}$, et al. A genome-wide association study of type 2 diabetes in Finns detects multiple susceptibility variants. Science 2007; 316:1341-1345.

20. Diabetes Genetics Initiative of Broad Institute of Harvard and MIT, Lund University, and Novartis Institutes of BioMedical Research, Saxena R, Voight BF, Lyssenko V, Burtt NP, de Bakker PI, et al. Genome-wide association analysis identifies loci for type 2 diabetes and triglyceride levels. Science 2007; 316:1331-1336.

21. Wellcome Trust Case Control Consortium. Genome-wide association study of 14,000 cases of seven common diseases and 3,000 shared controls. Nature 2007;447:661-678.

22. Cho YM, Kim TH, Lim S, Choi SH, Shin HD, Lee HK, et al. Type
2 diabetes-associated genetic variants discovered in the recent genome-wide association studies are related to gestational diabetes mellitus in the Korean population. Diabetologia 2009;52:253-261.

23. Lauenborg J, Grarup N, Damm P, Borch-Johnsen K, Jørgensen T, Pedersen $\mathrm{O}$, et al. Common type 2 diabetes risk gene variants associate with gestational diabetes. J Clin Endocrinol Metab 2009;94:145-150.

24. Ng MC, Park KS, Oh B, Tam CH, Cho YM, Shin HD, et al. Implication of genetic variants near TCF7L2, SLC30A8, HHEX, CDKAL1, CDKN2A/B, IGF2BP2, and FTO in type 2 diabetes and obesity in 6,719 Asians. Diabetes 2008;57:2226- 2233.

25. Kwak SH, Kim TH, Cho YM, Choi SH, Jang HC, Park KS. Polymorphisms in KCNQ1 are associated with gestational diabetes in a Korean population. Horm Res Paediatr 2010;74: 333-338.

26. Kwak SH, Park BL, Kim H, German MS, Go MJ, Jung HS, et al. Association of variations in TPH1 and HTR2B with gestational weight gain and measures of obesity. Obesity (Silver Spring) 2012;20:233-238.

27. Kwak SH, Kim SH, Cho YM, Go MJ, Cho YS, Choi SH, et al. A genome-wide association study of gestational diabetes mellitus in Korean women. Diabetes 2012;61:531-541.

28. Morris AP, Voight BF, Teslovich TM, Ferreira T, Segrè AV, Steinthorsdottir V, et al. Large-scale association analysis provides insights into the genetic architecture and pathophysiology of type 2 diabetes. Nat Genet 2012;44:981-990.

29. Scott RA, Lagou V, Welch RP, Wheeler E, Montasser ME, Luan $\mathrm{J}$, et al. Large-scale association analyses identify new loci influencing glycemic traits and provide insight into the underlying biological pathways. Nat Genet 2012;44:991-1005.

30. Lambrinoudaki I, Vlachou SA, Creatsas G. Genetics in gestational diabetes mellitus: association with incidence, severity, pregnancy outcome and response to treatment. Curr Diabetes Rev 2010;6:393-399.

31. Meigs JB, Shrader P, Sullivan LM, McAteer JB, Fox CS, Dupuis $\mathrm{J}$, et al. Genotype score in addition to common risk factors for prediction of type 2 diabetes. N Engl J Med 2008;359:22082219.

32. Lyssenko V, Jonsson A, Almgren P, Pulizzi N, Isomaa B, Tuomi $\mathrm{T}$, et al. Clinical risk factors, DNA variants, and the development of type 2 diabetes. N Engl J Med 2008;359:2220-2232. 